\title{
Identification of the Infrared Spectra Image using Improved SURF Method
}

\author{
Yuping Sun ${ }^{1, a}$, Xiaojia Jiang, ${ }^{1, b}$, Qiang $\mathrm{Hu}^{1, \mathrm{c}}$, Mingfei $\mathrm{Wu}^{1}$, \\ Guozhu Zhang1, Yiting Lv' ${ }^{1}$ and Lei Yang ${ }^{2}$ \\ ${ }^{1}$ Institute of NBC Defense, 102205, Beijing, China \\ ${ }^{2}$ Unit 66321, 102112, Beijing, China

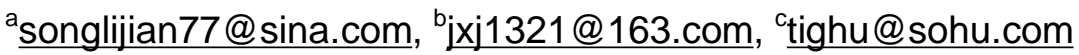

\begin{abstract}
Keywords: bilateral filter, canny method, Morphological operation, SURF
Abstract. Applying the bilateral filter, Canny method to detect the edge of the image, the Morphological operation to modify the result, we proposed an improved SURF algorithm. The improved SURF algorithm can effetely handle the image match problems where the image undergoes noise and texture blurry. We utilize the improved SURF algorithm to deal with the infrared image recognition problem and obtain a good result.
\end{abstract}

\section{Introduction}

The SURF algorithm is the speed up and robust SIFT [1] (Scale-invariant feature transform) algorithm. SIFT algorithm is adapted to image rotation, shift, and illumination changes, however the cost of the calculation of the method is very high and the data to be processed is big. SURF [2] (Speed Up Robust Feature) algorithm is the improved SIFT algorithm, and it can offer a better performance than SIFT, that is, it is more efficient and robust [3]. When the SURF algorithm is used to deal with infrared image recognition problem, we find that when the image undergoes noise and texture blurry, the traditional SURF method cannot offer a good result. So in this paper, based on the traditional SURF method, we proposed an improved SURF method. We utilize the improved SURF method to deal with the infrared image recognition problem, and get a good result. The proposed algorithm is robust and the speed of the improved SURF method outperforms the SIFT algorithm.

\section{SURF Algorithm}

The SURF algorithm consists of three parts: key-points detection, descriptor generation and keypoints matching.

\section{Key-points detection}

In the key-points detection part, we construct the image scale space namely the Gaussian pyramid firstly. Then we use the Gaussian pyramid to find the interest points in different scales. In order to speed up the generation the of interest points, we need to calculate the integral image.

The aim of using the integral image is to speed up the calculation of the Gaussian pyramid. As a result, the task of calculating the pixel sum of a rectangular region is reduced to four operations, and the calculation of first-order Haar wavelet response can be completed within six operations. Using the integral image can calculating the pixel sum of a rectangular region efficiently.

Constructing the image scale space (the Gaussian pyramid) is mainly used to find the key-points of the target in different scales. The SURF algorithm relies on the changing of the image size to create the Gaussian pyramid, so it can reduce the complexity of the calculation.

In SURF algorithm, the key-points detection uses the Hessian matrix determinant value to judge whether a point is an extreme value point. Then for every extreme value point, non-significantly inhibit is utilized to locate the key-point precisely. In the non-significantly inhibit method, every 
extreme value point is compared with the adjacent 26 points in the image scale space. If the value of one point is greater than or less than the rest of the 26 points, the point is called a key point.

\section{SURF feature descriptor generation}

In order to generate the SURF feature descriptor, we need to determine the dominant orientations of each key point. Then we can construct the descriptor vector.

We use Haar wavelet response [4] to calculate the dominant orientations of each key point. Within the neighbourhood regions, setting the key point as the center of the circle, $6 \sigma$ as the radius, we calculate the Haar wavelet response in the $\mathrm{X}$ and $\mathrm{Y}$ direction respectively. The side length of the Haar wavelet is set to be $4 \sigma$. For every $60^{\circ}$ fan-shaped area, we calculate the vector sum of the Haar wavelet responses in this region. And the direction with the maximum vector sum is considered as the dominant orientation.

According to the dominant orientations, we can determine a square area, and the side length is $2 \sigma$. The square area is divided into $4 \times 4$ sub-areas. In each sub-areas, we calculated the Haar response in the dominant orientation $\mathrm{dx}$ and in the vertical orientation dy. Each sub-area can be represented by four values: $\sum d x, \sum d y, \sum|d x|, \sum|d y|$. Thus the number of all the eigenvalues is 64 . Normalizing the 64 values, we can get the Feature descriptor of the key point. SURF feature descriptor is not sensitive to image rotation, scale changes, illumination changes and contrast changes.

\section{Key-points matching}

Calculating the maximum value of two vector inner product, we can obtain the two best matching points. Set a threshold $\mathrm{T}_{1}$. Only if the maximum inner product is greater than the threshold $\mathrm{T}_{1}$, the two point can be considered successful matching. Set a threshold $\mathrm{T}_{2}$. Only if the number of matching point of two image is greater then $T_{2}$, the two image can be considered successful matching.

\section{Improved SURF method}

When the image undergoes noise and texture blurry, the traditional SURF method cannot offer a good result. In this paper, we proposed an improved SURF method which is more efficient, more robust than SIFT algorithm. Firstly, we use bilateral filter to process the image, and then Canny operator is utilized to detect the edge of the image. Morphological operation is adopted to modify the processed result. After all the above preprocessing steps, we use SURF algorithm to find the feature points near the edge and then do feature point matching. The feature points found by this method is more reliable, thus reducing the mismatch.

\section{Bilateral Filtering}

First, Bilateral Filtering is proposed by V.Aurich and J.Weule in the study of nonlinear gauss filter and be officially named by C.Tomasi and R.Manduchi [5]. Unlike the traditional low-pass filter, bilateral filter not only consider the gray similarity of the pixel neighborhood, but also consider the space adjacent relationship of the pixels. The traditional low-pass filter such as Gaussian filter only consider the gray similarity of the pixel neighborhood, as a result, the high frequency information such as edges and noise will be filtered inevitably, thus losing the edge information and leading to edge blurry phenomenon. Bilateral filter combine the space adjacent relation and the gray similarity relation nonlinearly, so it solves the edge-filtering problem in a good way. Let ${ }_{\text {BL }}$ represent the bilateral filter, thus the math descriptor of Bilateral filter as follows:

$$
H_{B L}\left(I_{x}\right)=\frac{1}{W_{x}} \sum_{y \in S} G_{\sigma_{d}}\left(\|x-y\| G_{\sigma_{d}}\left(I_{x}-I_{y}\right)\right.
$$

Where $W_{x}$ is defined as: 


$$
W_{x}=\sum_{y \in S} G_{\sigma_{i}}(\|x-y\|) G_{\sigma_{i}}\left(I_{x}-I_{y}\right)
$$

Where $x$ represents the location the current point, $y \in S$ represents the points in the region centered in point $\mathrm{x}$ and sized by $S * S ._{\mathrm{I}_{\mathrm{x}}}$ and $\mathrm{I}_{\mathrm{y}}$ are the pixel values in the corresponding positions respectively. $\|x-y\|$ represents the space distance. $G_{\sigma_{i}}$ represents the space adjacent function, which can effectively avoid the influence of the abrupt changes of the pixel values. The space adjacent function $G_{\sigma_{i}}$ and the gray similarity function $G_{\sigma_{r}}$ are Gaussian functions using Euclidean distance, and they are defined as follows:

$$
G_{\sigma_{d}}=e^{\left.-\frac{1}{2} \frac{d(x, y)}{\sigma_{d}}\right)^{2}}
$$

Where $d(x, y)$ represents the Euclidean distance of two pixels in the image. $\sigma_{d}$ represents the Gaussian standard deviation.

$$
G_{\sigma_{r}}=e^{\left.-\frac{1}{2} \frac{\sigma(I(x), I(y))}{\sigma_{r}}\right)^{2}}
$$

Where $\sigma(I(x), I(y))$ represents the Gray difference of two pixels in the image and $\sigma_{\mathrm{r}}$ represents the Gaussian standard deviation.

Here $\sigma_{d}$ and $\sigma_{\mathrm{r}}$ play key roles. They control the filtering effect via limiting the relative spatial location and gray changes. So they can balance the performance of the bilateral filter well.

Bilateral filter combines the space adjacent relation with the gray similarity relation nonlinearly, thus controlling the performance of the filter in a good way. As shown in figure 1, fig. (a) is the source image without filtering. The ladder in the image represents the edge information, and the small fluctuations are noise points. Fig. (b) is the image processed by Gaussian filter. Most noise point are filtered, however this method loses the edge information. Fig. (c) is the image processed by bilateral filter. It is clear that this method filters out the noise points and also reserves the edge information. The experiment demonstrates that the advantage of the bilateral filter is it can filter out most of the noise and retain the edge information at the same time.

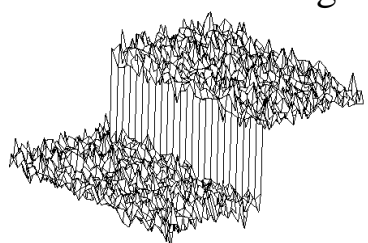

(a)

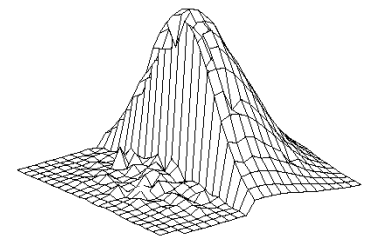

(b)

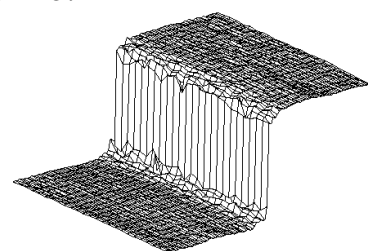

(c)

Fig.1: Bilateral filter

As shown in figure2, fig(a) is the input of the experiment. In the source image, many noises are distributed in the hat and in the face of Lena. Fig.(b) and Fig.(c) are the results of Gaussian lowpass filter and the bilateral low-pass filter respectively. For fair comparison, all the parameters of the two filters are the same. In Fig.(b), the noise points are filtered out, however the edge information also lost, so the image is blurry. In Fig.(c), processed by the bilateral filter, most noises distributed in the hat and in the face of Lena are filtered out, so the internal information of the image is processed very smooth, meanwhile the edge of the image is still very clear. The experiment demonstrates that compared to the traditional low-pass filter, the bilateral filter can filter out the noise and remain the edge information at the same time.

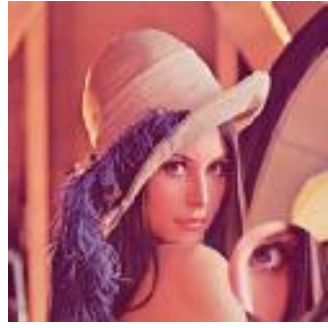

(a)

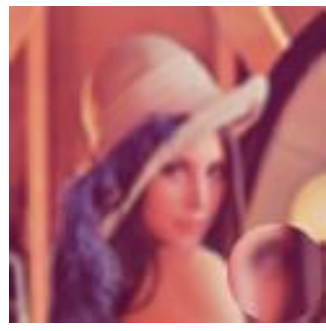

(b)

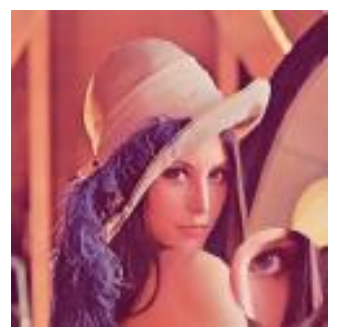

(c)

Fig.2: The result of bilateral filter 
The image processed by bilateral filter becomes very smooth, and the edge information is remained at the same time. So we can draw conclusion that compared to the traditional low-pass filter, the bilateral filter can de-nosing and remain the edge information.

\section{Edge Detection}

The edge is the position where the pixel values change dramatically in the image. As one of the basic characteristics of the image, it exists between the background and the foreground of the image. The edge is not sensitive to the changes in gray, so it has a unique geometric characteristic. It is an important feature in the image segmentation and image registration problems. There are many image edge detection operators and the commonly used edge detection operations include the Sobel operator [6], the Laplace operator [7], the Canny operator [8] and so on.

Utilizing the signal to noise ratio and the positioning product, the Canny operator is an optimal approximation operator. The operator first uses the Gaussian filter to smooth the image which will lead to edge diffusion phenomenon. To solve this problem, The Canny operator use the maximum inhibition to reduce the impact of non-edge pixels. Thus it can remove the non-edge pixels in a good way so as to preserve the protruding edges. Calculate the first derivative of the smooth image and then calculate the gradient magnitude and gradient direction via the first-order partial derivatives finite difference. Since Canny operator utilize the dual-threshold to detect the edge, it can eliminating non-edge information very well. So the detected edge can achieve a better result.

Compared to other operators, Canny operator has the following advantages: Canny operator can correctly detect more edges, and thus the detected edge has a high location degree; Canny operator has a good noise immunity, so it can inhibit the false contour very well. The edges detected by Canny operator are full relatively, so interrupted edge phenomenon occurs rarely. Meanwhile, Canny operator can detect the weak edge very well.

As shown in figure3, fig.(a) is the input of the experiment. Fig.(b) is the result of the Bilateral filter. Fig.(c) is the result of edge detection processed by Sobel operator. Fig.(d) is the result of edge detection processed by Canny operator. It is clear that the noise points in Fig.(d) is less than in Fig.(b) and Fig.(c), and the result of the detection in Fig.(d) outperforms results in Fig.(b) and Fig.(c).

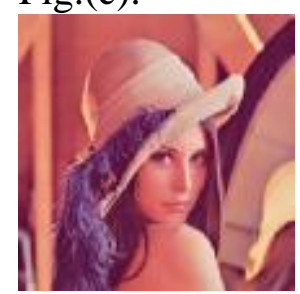

(a)

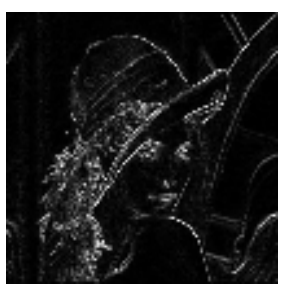

(b)

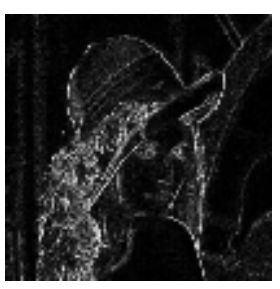

(c)

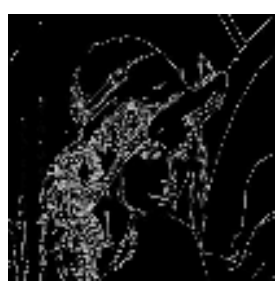

(d)

Fig.3: The result of edge detection

\section{Edge Detection}

Before Based on mathematical morphology set theory, Morphological processing is a binary image processing method. The main operations include expansion, corrosion, the open operation and close operation. Expansion is used to enlarge the target, while corrosion is used to make the target smaller. Expansion operation can bridge some small cracks in the image, and corrosion operation can remove the irrelevant details in the binary image. The open operation combines the expansion and the corrosion, which firstly corrode the image using the structuring element and then expanse the image. The close operation is the dual operation of the open operation which firstly expanse the image using the structuring element and then corrode the image. The open operation can break the narrow intermittent area and eliminate small protrusions, while the close operation can eliminate the narrow discontinuities and the elongated gap area and fill up the broken in the contours. 


\section{Comparison Results of Feature Point Detection}

As shown in figure 4, the image in fig. (a) is the result of feature point detection using the traditional SURF method, while the image in fig. (b) is the result of feature point detection using the improved SURF method. The comparison result demonstrated that the improved SURF method can detect more feature points, so it is adapted to the identification of the infrared spectra image.

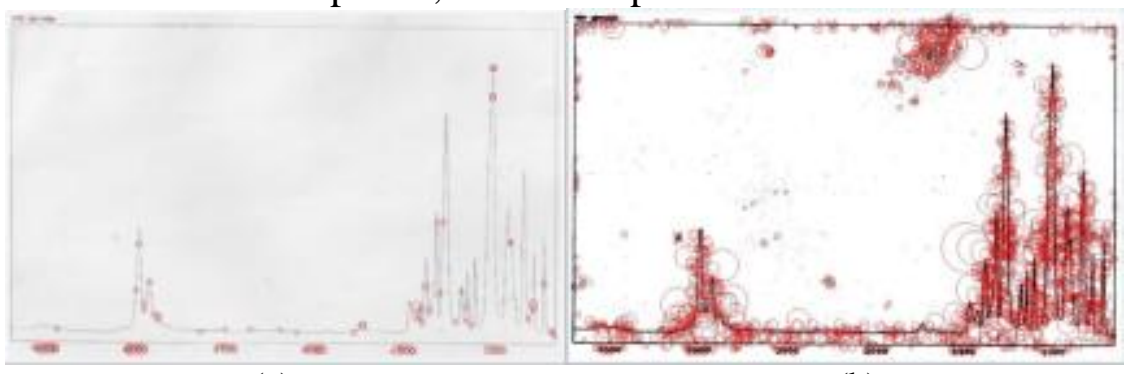

(a)

(b)

Fig.4: The comparison results of the feature point detection using the traditional SURF method (a) and the improved SURF method (b).

The simulation results demonstrate that the improved SURF method can effectively handle the image matching problems especially for image undergoes noise interference and texture blurring. The improved SURF method is more robust and stable than SIFT. Moreover, it achieves lower computation complexity and faster running speed.

\section{References}

[1] Lowe D G. Distinctive image features from scale-invariant keypoints[J]. International journal of computer vision, 2004, 60(2): 91-110.

[2] Bay H, Tuytelaars T, Van Gool L. Surf: Speeded up robust features[M]//Computer VisionECCV 2006. Springer Berlin Heidelberg, 2006: 404-417.

[3] Juan L, Gwun O. A comparison of sift, pca-sift and surf[J]. International Journal of Image Processing (IJIP), 2009, 3(4): 143-152.

[4] Huang J, Mumford D. Statistics of natural images and models[C]//Computer Vision and Pattern Recognition, 1999. IEEE Computer Society Conference on. IEEE, 1999, 1.

[5] Tomasi C, Manduchi R. Bilateral filtering for gray and color images[C]//Computer Vision, 1998. Sixth International Conference on. IEEE, 1998: 839-846.

[6] Ying-Dong Q, Cheng-Song C, San-Ben C, et al. A fast subpixel edge detection method using Sobel-Zernike moments operator[J]. Image and Vision Computing, 2005, 23(1): 11-17.

[7] Perona P, Malik J. Scale-space and edge detection using anisotropic diffusion[J]. Pattern Analysis and Machine Intelligence, IEEE Transactions on, 1990, 12(7): 629-639.

[8] LIN H, ZHAO C, SHU N. Edge detection based on Canny operator and evaluation [J]. Journal of Heilongjiang Institute of Technology, 2003, 2: 001. 